\title{
Fully 3D Uniform Resolution Transmission microPET Image Reconstruction ${ }^{1}$
}

\author{
${ }^{\dagger}$ Bing Bai, ${ }^{\ddagger}$ Patrick Chow, ${ }^{\ddagger}$ Arion Chatziioannou and ${ }^{\dagger}$ Richard M. Leahy \\ ${ }^{\dagger}$ Signal and Image Processing Institute, University of Southern California, Los Angeles, CA 90089 \\ ${ }^{\ddagger}$ Crump Institute for Molecular Imaging, University of California, Los Angeles, CA 90095
}

\begin{abstract}
MAP (maximum a posteriori) reconstructions of transmission images produce more accurate ACFs (attenuation correction factors) than smoothed division of blank and transmission scans and analytical methods. Also, for highly speciPc tracers, transmission images can provide useful anatomical cues for use in localizing structures and image coregistration. The resolution of MAP images reconstructed with a spatially invariant smoothing prior is known to be nonuniform. Spatially variant smoothing parameters are used here to achieve uniform resolution throughout the transmission image. We also investigate the relationship between resolution and the global smoothing parameter through computer simulations. The resulting calibration curve can be used to select the smoothing parameter to achieve a desired spatial resolution. The tractability and feasibility of our method is demonstrated through an application to phantom transmission data collected using a Concorde P4 microPET system.
\end{abstract}

\section{INTRODUCTION}

Attenuation correction is important for quantitative PET studies. Attenuation correction factors (ACFs) are computed either by smoothed division of a blank and transmission scan or through reconstruction and reprojection of the attenuation image. In some cases reprojection is preceded by segmentation of the transmission image to ameliorate the effects of noise and scatter on the computed ACFs. Traditionally transmission scans are performed in 2D modes using septa. With the emergence of septa-less 3D PET scanners [1], it is desirable to have a fully $3 \mathrm{D}$ transmission reconstruction algorithm.

In our previous work we developed MAP reconstruction algorithms for 2D transmission scans [2] and 3D emission scans [3] which have been successfully applied to clinical and small animal PET scanners. Here we extend our work to a fully 3D transmission reconstruction method. The proposed method has been applied to Concorde P4, a high resolution small animal microPET scanner without septa. Transmission scans can be performed in either coincidence or singles modes using a spirally rotating Ge-68 point source. For this study we used singles data, but our method is also applicable to coincidence transmission scans.

Images reconstructed with MAP algorithms are known to have nonuniform resolution and this problem has been addressed [4]. Here we use space-variant smoothing parameters to compensate for it. Simulations show that the resolution of reconstructed transmission images are approximately uniform.

\footnotetext{
${ }^{1}$ This work was supported by the National Institute of Biomedical Imaging and Bioengineering under Grant No. R01 EB00363.
}

The next step is to choose a global smoothing parameter to adjust the resolution of the transmission image. This technique avoids artifacts in attenuation corrected emission images caused by oversmoothed transmission data [5], [6].

\section{Methods}

\section{A. Statistical Model}

Transmission scan data can be modeled as a collection of independent Poisson random variables with mean $\bar{y}_{i}$ given by

$$
\bar{y}_{i}=\frac{\tau_{t}}{\tau_{b}} \bar{b}_{i} \exp \left(-[\mathbf{P} \mathbf{x}]_{i}\right)+\bar{r}_{i}
$$

where $\mathbf{x} \in \mathbf{R}^{M \times 1}$ is the attenuation coefPcient at each voxel, $\overline{\mathbf{b}} \in \mathbf{R}^{N \times 1}$ is the mean of the blank scan sinogram, which can be approximated by directly using a blank scan of sufPcient duration, $\tau_{t}$ is the transmission scan duration, $\tau_{b}$ is the blank scan duration, $\mathbf{P} \in \mathbf{R}^{N \times M}$ is the geometric projection matrix and $\overline{\mathbf{r}} \in \mathbf{R}^{N \times 1}$ is the mean value of background counts. For coincidence scans, the background counts include scatters and contamination due to randoms and emission. For singles scans, there are no randoms but some LSO background is introduced.

In MAP reconstructions, our objective function is the $\log$ posterior function:

$$
\phi(\mathbf{x}, \mathbf{y})=L(\mathbf{y} \mid \mathbf{x})-\beta U(\mathbf{x})
$$

where $\mathbf{y} \in \mathbf{R}^{N \times 1}$ is the measured transmission sinogram, $U(\mathbf{x})$ is the prior function, $\beta$ is a smoothing parameter controlling the smoothness of the image and $L(\mathbf{y} \mid \mathbf{x})$ is the Poisson loglikelihood function given by:

$$
L(\mathbf{y} \mid \mathbf{x})=\sum_{i=1}^{N} y_{i} \log \bar{y}_{i}-\bar{y}_{i}
$$

\section{B. Preconditioned Conjugate Gradient (PCG)}

We use the PCG algorithm to maximize the posterior function (2) obtained in the previous section. The preconditioned Polak-Ribiere form of the conjugate gradient algorithm is:

$$
\begin{array}{r}
\mathbf{x}^{(n+1)}=\mathbf{x}^{(n)}+\alpha^{(n)} \mathbf{a}^{(n)} \\
\mathbf{a}^{(n)}=\mathbf{d}^{(n)}+\beta^{(n-1)} \mathbf{a}^{(n-1)} \\
\mathbf{d}^{(n)}=\mathbf{C}^{(n)} \mathbf{g}^{(n)} \\
\beta^{(n-1)}=\frac{\left(\mathbf{g}^{(n)}-\mathbf{g}^{(n-1)}\right)^{\prime} \mathbf{d}^{(n)}}{\mathbf{g}^{(n-1)^{\prime}} \mathbf{d}^{(n-1)}}
\end{array}
$$


where $\mathbf{g}^{(n)}$ is the gradient vector of the log posterior function $\phi(\mathbf{x}, \mathbf{y})$ at $\mathbf{x}=\mathbf{x}^{(n)}$, stepsize $\alpha^{(n)}$ is found by using a NewtonRaphson line search. $\mathbf{C}^{(n)}$ is the preconditioner, here we use the preconditioner proposed in [2]:

$$
\mathbf{C}^{(n)}=\operatorname{diag}\left\{\frac{x_{k}^{(n)}}{\sum_{i} p_{i k}}\right\}
$$

\section{Resolution}

For MAP estimators, the local impulse response (LIR) can be used to analyze the resolution of reconstructions [4]. The local impulse response of the $j^{\text {th }}$ voxel is dePned as:

$$
l^{j}(\mathbf{x})=\lim _{\delta \rightarrow 0} \frac{E\left(\hat{\mathbf{x}}\left(\mathbf{y}\left(\mathbf{x}+\delta \mathbf{e}_{j}\right)\right)\right)-E(\hat{\mathbf{x}}(\mathbf{y}(\mathbf{x})))}{\delta}
$$

As shown in [4], the local impulse response can be approximated using a truncated series expansion with an expression dependent on the Fisher information matrix. The Fisher information matrix for the likelihood in (3) is given by

$$
\mathbf{F}=\mathbf{P}^{\prime} \operatorname{diag}\left\{u_{i}\right\} \mathbf{P}
$$

For emission reconstruction, $u_{i}=\frac{1}{\bar{y}_{i}}$, while for transmission, $u_{i}=\left(\bar{y}_{i}-r_{i}\right)\left(1-\frac{r_{i} y_{i}}{\bar{y}_{i}^{2}}\right)$.

For space-invariant priors, the local impulse response can be approximated by [7]

$$
l^{j}(x) \approx[\mathbf{F}(j)+\beta \mathbf{R}(j)]^{-1} \mathbf{F}(j) \mathbf{e}_{j}
$$

where $\mathbf{F}(j)$ is a local invariant approximation of the Fisher information and $\mathbf{R}(j)$ is a local invariant approximation of the second derivative of the prior energy function; $\mathbf{e}_{j}$ is the $j^{\text {th }}$ unit vector.

Here we use the contrast recovery coefficient (CRC) as a measure of image resolution [7], which we dePned as $C R C_{j}=$ $l_{j}^{j}(x)$, i.e. the height at voxel $j$ of the local impulse response at voxel $j$. Our goal is to have constant CRC values throughout the image. For 3D PET systems, the CRC for the $j^{t h}$ voxel can be approximated by:

$$
C R C_{j} \approx \frac{1}{N} \sum_{i} \frac{\lambda_{i}(j)}{\lambda_{i}(j)+\beta \kappa_{j}^{-2} \mu_{i}}
$$

where $\lambda_{i}(j)$ depends on the geometric matrix $\mathbf{P}, \mu_{i}$ depends on the prior function, and $\kappa_{j}$ is the $j^{\text {th }}$ diagonal element of the Fisher information matrix.

We can see from the above approximation of CRC that the resolution of images reconstructed using the pure form of the MAP technique is not uniform. This property is well known, and modipcation to the smoothing parameter in order to achieve uniform resolution has been proposed [4]. However the method in [4] was only evaluated for the 2D case, where the system is approximately shift-invariant. An extension of this idea to 3D is described in [7] in which the Fisher information, and hence the resolution, is highly spatially variant due to large differences in sensitivity throughout the image volume. In this paper we follow [7] and also note that in the resolution analysis mentioned above, the only difference between transmission and emission scans is the form of the Fisher information matrix [8].

From (9) we can see that the data-dependency of the CRCs comes from the $\kappa_{j} \tilde{\Theta}$ only. For a particular imaging system and smoothing function, the $\lambda_{i}(j) \tilde{\Theta}$ are spatially varying constants. To achieve a spatially invariant CRC we must therefore choose the smoothing parameter $\beta$ as a function of the Fisher information so that the product $\eta_{j}=\beta \kappa_{j}^{-2}$ gives the desired CRC in (9) at each voxel $j$. The problem is complicated by the fact that spatial interactions in the smoothing function cause coupling between the spatially variant smoothing parameters. To account for this we write the quadratic smoothing function in the following way:

$$
\beta \mathbf{U}(\mathbf{x})=\frac{1}{2} \beta \sum_{j=1}^{N} \sum_{k \in N_{j}, k>j} \delta_{j k} \phi_{j} \phi_{k}\left(x_{j}-x_{k}\right)^{2}
$$

where $\delta_{j k}$ is the reciprocal of the Euclidean distance between voxels $j$ and $k, \phi_{j}$ and $\phi_{k}$ are space-variant smoothing parameters, $\beta$ is a global scale factor, and $N_{j}$ is the set of neighboring voxels of voxel $j$.

To achieve uniform CRCs, a least-squares approach for solving the following set of equations for $\phi$ was proposed in [7]:

$$
\sum_{k \in N_{j}} \delta_{j k} \phi_{j} \phi_{k}=\eta_{j} \kappa_{j}^{2} \sum_{k \in N_{j}} \delta_{j k}
$$

where $\eta_{j}$ is the value found from lookup tables that gives the same CRC for each voxel $j$. In practice, as an approximate solution, assuming the $\kappa_{j} \widetilde{\Theta}$ are smooth and the relationship between CRC and $\beta$ is shift-invariant ${ }^{2}$, we simply use a Prst order approximation $\phi_{j}=\kappa_{j}$.

To determine the actual CRC for the reconstructed image, we calibrate the relationship between the global parameter $\beta$ and CRC in simulations and then use a look-up table to achieve the desired CRC. We compute this curve for both emission and transmission reconstructions noting that the only difference in the resolution analysis between the two cases is in the diagonal elements of the Fisher information matrix, $\kappa_{j}$. Once we use space-variant smoothing parameters to cancel the effect of $\kappa_{j} \tilde{\mathrm{O}}$, the resolution analysis is, to a Prst order approximation, the same for transmission and emission images.

To check the resolution uniformity of the proposed method, we simulated a uniform cylinder with a cold chamber. The simulated attenuation coefPcient is $0.095 / \mathrm{cm}$ inside the cylinder

\footnotetext{
${ }^{2}$ We found that the relationship between CRC and $\beta$ for the range of CRC $<0.1$ is approximately shift invariant; see for example, Fig 1a in [7].
} 
and $0.03 / \mathrm{cm}$ in the cold chamber. Fig. 1 shows one plane from the simulated phantom. The white points are those for which the LIRs were computed in Fig. 1. Assuming that the mean of the estimator equals the estimate obtained from noiseless data, we can compute the local impulse response function from two noiseless reconstructions as suggested in [4].

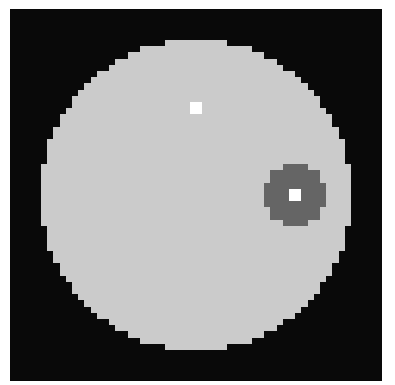

Figure 1: One plane of the simulated phantom
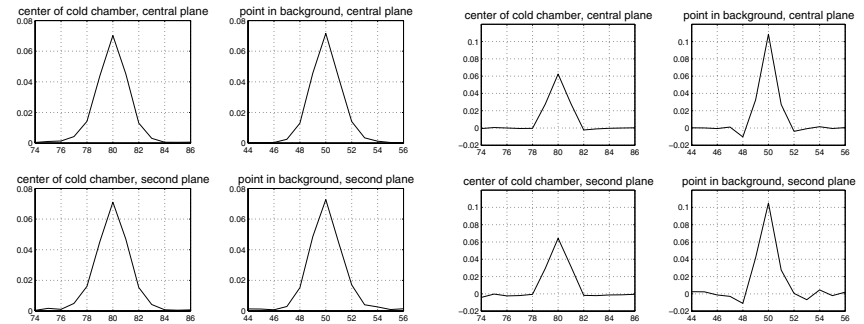

Figure 2: Radial plots of LIR for four points with space-variant smoothing parameters (left two columns) and without space-variant smoothing parameters (right two columns).

Fig. 2 shows the radial proPle of the LIR for four points, two in the central plane and two in the second of 63 transaxial planes. Within each plane the two points are selected such that they have the same radial distance from the center of the cylinder with one of them being at the center of the cold region. The uniformity of the resolution is clearly seen. For comparison the corresponding proPles when the space-variant smoothing parameter was not used are also shown. It is obvious that they vary with the position of the point.

The simulated phantom was also used to compute the CRC curve. The PET system simulated here is a simpliped Concorde R4 system, which has the same transaxial geometry but only 4 detector rings. We computed the CRC at the center of the scanner as a function of the global smoothing parameter for both transmission and emission simulations. The resulting curves are shown in Fig. 3.

In [5] it was shown that the resolution mismatch between the emission and transmission data can cause artifacts in the attenuation corrected emission images. Similar artifacts are also observed in statistical reconstruction methods [6]. A resolution-matching method was proposed in [5]. The proposed method works well with analytical reconstruction methods. For statistical reconstruction methods, the situation is more complicated because the resolution of transmission images are usually nonuniform. When we have uniform

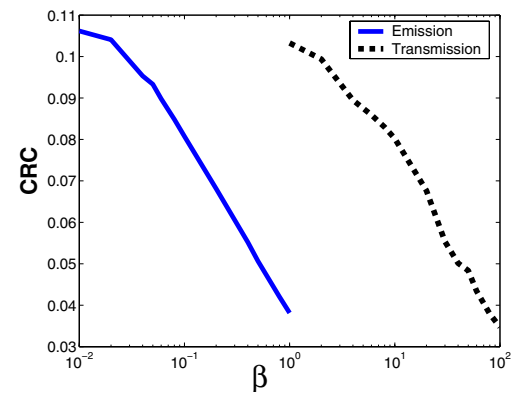

Figure 3: Emission and transmission CRC curves for the point located at scanner center

resolution due to space-variant smoothing parameters, we can choose an appropriate global smoothing parameter to achieve a desired resolution, thus avoiding these artifacts. The transmission image should be neither too smooth, thus causing artifacts, nor too noisy, otherwise noise will be introduced into the Pnal emission image. Optimization of the resolution in transmission images to achieve the best quantitative accuracy in emission images is not explored here, but the CRC studies of transmission and emission data should provide a useful guide to selection of smoothing parameters to minimize errors propagating from transmission to emission images.

\section{RESUlTS}

\section{A. Computation}

Sinogram data from the Concorde P4 microPET scanner was rebinned with a span of 3 to $703192 \times 168$ sinograms. The transmission image was generated by running our PCG algorithm for 30 iterations, each of which takes around 4 minutes on a $1.7 \mathrm{GHz}$ Pentium IV dual-CPU PC which has 1GB RAM.

\section{B. Phantom Study}

A $5 \mathrm{~cm}$ diameter cylindrical phantom containing two smaller cylindrical chambers was scanned. For the emission scan, only the large background chamber was Plled with a water solution of F-18 $(280 \mu \mathrm{Ci})$. One of the two smaller chambers was plled with water while the other was left empty (air). After allowing enough time for decay of the emission activity, $(>10$ half-lives) a transmission scan was initiated with a $0.5 \mathrm{mCi}$ positron emitting point source spiraling through the FOV. We used our $3 \mathrm{D}$ transmission reconstruction code to generate the transmission image, which was then forward projected and used in the emission reconstruction. The transmission image and the attenuation corrected emission images are shown in Fig. 4.

\section{Conclusion}

In this paper, a fully 3D MAP reconstruction algorithm for transmission is implemented and applied to a Concorde P4 small animal microPET scanner. Preliminary results from simulation and cylindrical phantom scans show that 

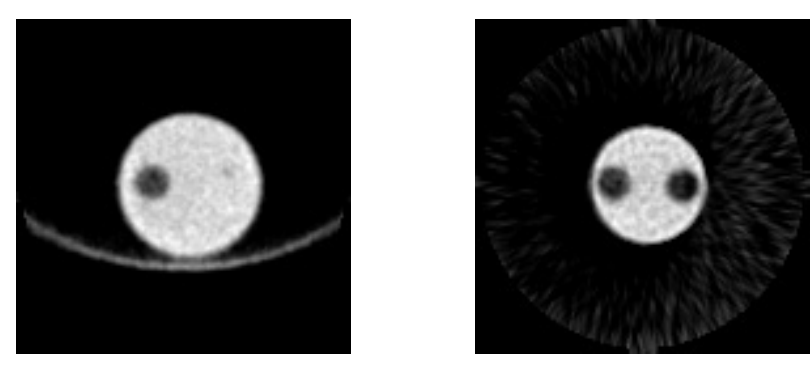

Figure 4: Central plane of the reconstructed phantom image: Transmission image (left) and Emission image (right)

the proposed method is feasible. By using a space-variant smoothing parameter, approximate uniform resolution is achieved. We can calibrate the resolution of transmission and emission images as a function of a global smoothing parameter $\beta$, the result of which can be used to avoid the artifacts caused by resolution mismatch and to optimize the Pnal emission image.

\section{REFERENCES}

[1] J.S. Karp, A.J. Becher, S. Matej and P.E. Kinahan, Data Processing and Image Reconstruction Methods for The Head PENN-PET Scanner, IEEE Trans. Nucl. Sci., Vol 45, pp. 1144Đ1151,Jun 1998

[2] E.U. Mumcuoglu, R.M. Leahy, S.R. Cherry and Z. Zhou, Fast Gradient-based Methods for Bayesian Reconstruction of Transmission and Emission PET Images, IEEE Trans. Med. Imaging, Vol 13, pp. 687Đ701,1994

[3] J. Qi, R.M. Leahy, S.R. Cherry, A. Chatziioannou and T.H. Farquhar, High-Resolution 3D Bayesian Image Reconstruction Using the MicroPET Small-Animal Scanner, Phys. Med. Biol. 43, pp. 1001Đ1013,1998

[4] J.A. Fessler and W.L. Rogers, Spatial Resolution Properties of Penalized-Likelihood Image Reconstruction: Space-Invariant Tomographs, IEEE Trans. Image Processing, Vol 5, pp. 1346Đ1358,Sep 1996

[5] A. Chatziioannou and M. Dahlbom, Detailed Investigation of Transmission and Emission Data Smoothing Protocols and Their Effects on Emission Images, IEEE Trans. Nucl. Sci. , Vol 43, pp. 290Đ294,Feb 1996

[6] H. Erdogan, Statistical Image Reconstruction Algorithms Using Paraboloidal Surrogates for PET Transmission Scans, Ph.D Thesis, Univ. Mich., Ann Arbor, 1999

[7] J. Qi and R.M. Leahy, Resolution and Noise Properties of MAP Reconstructions in Fully 3D PET, IEEE Trans. Med. Imaging, Vol 19, pp. 493Đ506, May 2000

[8] J.A. Fessler, Resolution properties of regularized image reconstruction methods, Tech. Rep. 297, Commun. and Signal Processing Lab., Dept. of Elect. Eng. Comput. Sci, Univ. Mich., Ann Arbor, Aug 1995 